\title{
Evolución histórica de la mortalidad de los pacientes internados en el Centro Nacional de Quemados entre 1995 y 2017
}

\author{
Ignacio Álvez*, Martín Angulo*, Ignacio Aramendi†, Agustín Carámbulał,
} Julio Cabrera§, Gastón Burghi†

\section{Resumen}

Introducción: la mortalidad de los pacientes con injuria térmica ha descendido a nivel mundial. Este hecho se ha relacionado en parte con la creación de centros especializados en el tratamiento de esta patología. En nuestro medio no existen estudios recientes que evalúen la evolución de la mortalidad en este tipo de centros.

Objetivo: describir las características de la población asistida y la evolución de la mortalidad en el Centro Nacional de Quemados. Material y método: estudio transversal, observacional, descriptivo. Se incluyeron todos los pacientes ingresados con diagnóstico de quemadura cutánea o de injuria inhalatoria desde la inauguración del centro en 1995 hasta el 31 de diciembre de 2017. Resultados: ingresaron en el período de estudio 3.050 pacientes quemados, $25 \%$ presentaron una superficie corporal quemada superior o igual a 20\%. Existió un predominio de pacientes jóvenes (más de $60 \%$ menores de 50 años) y de sexo masculino (62\%). El agente causal más frecuente fue el fuego directo (71\%), seguido por líquidos calientes $(9 \%$ ) y electricidad (5\%). El $43 \%$ de los pacientes requirió asistencia ventilatoria mecánica, y la estadía media en el centro fue de 17 días. La mortalidad global fue de 19,7\%, similar a la esperada de acuerdo a scores de severidad específicos. La mortalidad de los pacientes más graves ha descendido desde la apertura del centro.

Conclusiones: nuestro centro asiste predominantemente a pacientes jóvenes, con siniestros que involucran al fuego como el principal agente causal. La mortalidad es acorde a la esperada de acuerdo a los scores de severidad, destacándose un descenso continuo de la misma a lo largo de los años.

Palabras clave: Quemaduras

Mortalidad

Unidades de quemados

Epidemiología

Key words: $\quad$ Burns

Mortality

Burn units

Epidemiology

\footnotetext{
* Asistente de Medicina Intensiva. Universidad de la República. Centro Nacional de Quemados.

† Profesor Adjunto de Medicina Intensiva. Universidad de la República. Centro Nacional de Quemados.

$\ddagger$ Residente de Medicina Intensiva.

$\S$ Profesor Agregado de Medicina Intensiva. Universidad de la República. Centro Nacional de Quemados.

Correspondencia: Dr. Gastón Burghi. Centro Nacional de Quemados. Hospital de Clínicas, Av. Italia s/n. Montevideo, Uruguay. Correo electróni-

co: burghig@gmail.com

Los autores declaran no tener conflictos de interés.

Recibido: 29/8/18

Aprobado: 5/11/18
} 


\section{Introducción}

La Sociedad Internacional de Quemaduras define una quemadura como la injuria de la piel o de cualquier tejido generado por una causa térmica. En este sentido, una quemadura ocurre cuando todas o algunas células de la piel o de otros tejidos son destruidos por fuego, líquidos calientes, sólidos calientes, radiación, electricidad o sustancias químicas.

Las quemaduras representan la cuarta causa de muerte entre los pacientes que sufren injurias traumáticas ${ }^{(1)}$. Anualmente más de 250 mil personas fallecen en el mundo a causa de quemaduras, ocurriendo la mayoría de estas muertes en países subdesarrollados.

Los diferentes países, de acuerdo a sus posibilidades económicas, presentan diferencias en el acceso a los cuidados de salud, y en esta patología específica, presentan diferencias en la posibilidad de acceso a centros especializados en el cuidado del paciente quemado ${ }^{(2)}$.

La mortalidad de los pacientes quemados ha presentado un descenso constante. Más allá del mayor conocimiento de las alteraciones fisiopatológicas que ocurren en el paciente quemado y de los avances terapéuticos, la experiencia en el manejo de esta patología, así como la mejor interacción de diferentes especialidades, se asocian a este incremento de la sobrevida ${ }^{(3,4)}$.

Nuestro país cuenta desde 1995 con el Centro Nacional de Quemados de adultos que nuclea a la mayoría de los pacientes adultos con quemaduras graves, injuria inhalatoria o quemaduras en áreas funcionales. En este centro trabajan en forma coordinada especialistas en medicina intensiva, anestesia, cirugía plástica, psiquiatría, psicología, así como un equipo de enfermería especializado en el tratamiento de este tipo de pacientes.

Si bien existen estudios que demuestran un descenso de la mortalidad en los centros especializados en el tratamiento de pacientes quemados, este tópico no ha sido evaluado en nuestro medio.

El objetivo de este estudio es describir las características de la población asistida y la evolución de la mortalidad en el Centro Nacional de Quemados.

\section{Metodología}

Este es un estudio transversal, observacional, descriptivo. El mismo fue aprobado por el Comité de Ética de la Investigación del Hospital de Clínicas. Se analizaron todos los pacientes ingresados al Centro Nacional de Quemados desde su inauguración en el año 1995 hasta el 31 de diciembre de 2017.

Se incluyeron todos los pacientes ingresados con diagnóstico de quemadura cutánea o de injuria inhalatoria. Se excluyeron del análisis los pacientes que ingresa- ron al centro con otros diagnósticos (dermopatías graves, escalpes u otro tipo de pacientes).

Para el análisis de los datos se recurrió al registro informático del centro, de donde obtuvimos los datos patronímicos de los pacientes, las características de los siniestros, el tipo de agente, la superficie quemada, la necesidad de ventilación mecánica invasiva y su duración, así como otras características de la asistencia.

\section{Definiciones}

Para establecer la severidad de los pacientes utilizamos el ABSI score (Abreviated Burn Score Index). Este es un score de severidad específicamente diseñado para población de pacientes quemados. El mismo toma en cuenta la superficie quemada, la edad, la presencia de injuria inhalatoria y el sexo. A mayor puntaje, mayor probabilidad de muerte ${ }^{(5)}$.

La mortalidad estandarizada por ABSI resulta del cociente entre la mortalidad real y la mortalidad predicha por el score.

\section{Análisis estadístico}

Se ingresaron los datos en planilla electrónica para su análisis posterior. Las variables cetegóricas se expresaron como porcentaje, mientras que las variables continuas se expresaron como mediana (percentiles $25 \%$ y $75 \%$ ). Las comparaciones de variables categóricas se hicieron con la prueba exacta de Fisher y de chi cuadrado (con la corrección de Yates cuando estuvo indicado). Las variables continuas se presentaron en la forma de media \pm desvío estándar o de mediana (intervalo intercuartil) y se compararon con la prueba t-Student o la prueba U de Mann-Whitney Rank-sum, según estuvo indicado. En todos los análisis se adoptó como nivel de significancia estadística un $\mathrm{p}$-valor $<0,05$ bilateral.

\section{Resultados}

\section{Características generales de la población y epidemiología del centro}

En el período analizado ingresaron 3.050 pacientes quemados, con predominio de sexo masculino (62\%), una edad de 42 años (28-60) y una superficie total quemada de $10 \%$ (3\%-22\%). Cabe destacar que más del $25 \%$ de los pacientes presentaron una superficie quemada superior o igual a 20\% (figura 1). La distribución por edades muestra un predominio de ingresos en los rangos etarios más jóvenes, destacándose que más del $60 \%$ de los ingresos fueron menores de 50 años (figura 2).

El agente causal de siniestros más frecuente fue el fuego directo (71\%), seguido por líquidos calientes en $9 \%$ y electricidad $5 \%$. Los siniestros causados por líqui- 


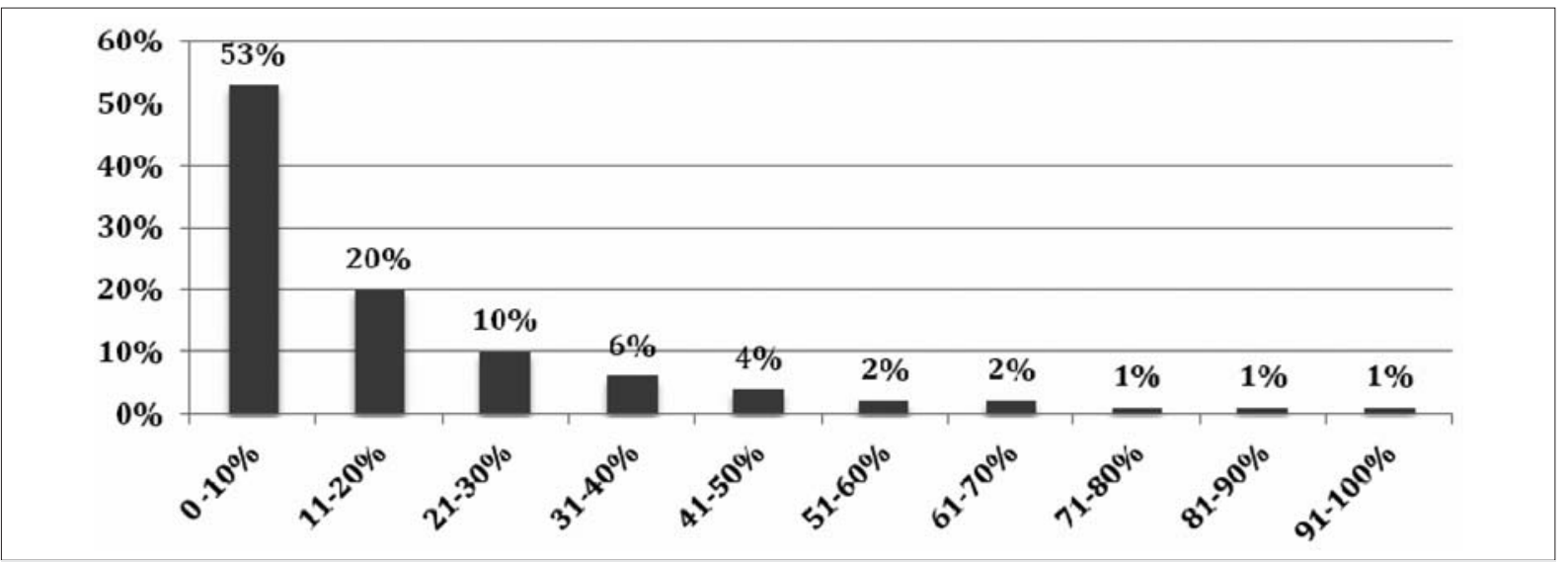

Figura 1. Distribución de los ingresos de acuerdo a la superficie corporal quemada.

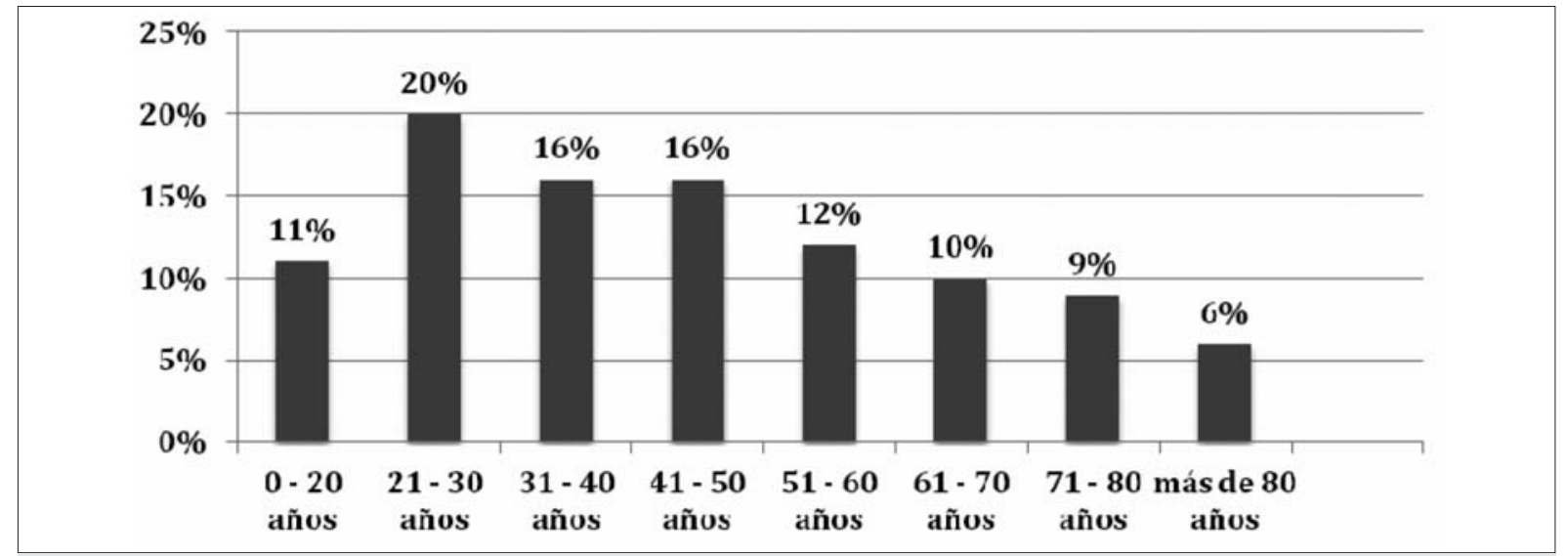

Figura 2. Distribución de los ingresos de acuerdo al rango etario.

dos calientes incrementan su frecuencia a partir de los 60 años (figura 3).

Los accidentes en el domicilio se presentaron en el $57 \%$ de los casos, seguidos por accidentes laborales e intentos de autoeliminación con $9 \%$ de los casos cada uno.

El $43 \%$ de los pacientes requirieron asistencia ventilatoria mecánica durante 6 días (3-16). La estadía en UCI fue de 17 días (7-32).

\section{Mortalidad estandarizada}

La mortalidad global fue de 19,7\%. Al analizar la mortalidad de acuerdo a la predicción del score ABSI (mortalidad real/mortalidad predicha por ABSI) encontramos una mortalidad estandarizada de 0,98 (figura 4).

\section{Evolución temporal de la mortalidad}

La evolución de la mortalidad analizada por quinquenios presenta dos comportamientos diferentes de acuerdo a la severidad de los pacientes. Los pacientes con un ABSI menor de 6 puntos no muestran variación de la mortalidad a lo largo del tiempo $(\mathrm{p}=0,54)$; en contraposición, los pacientes con ABSI de 6 puntos o más evidencian un descenso progresivo de la mortalidad $(p<0,001)$ (figura 5).

\section{Discusión}

Este estudio nos permitió conocer las características de los pacientes que ingresaron al Centro Nacional de Quemados desde su fundación así como la evolución de la mortalidad en el mismo.

El análisis de la epidemiología de nuestro centro nos permite observar que la mayor parte de los pacientes asistidos por quemaduras son pacientes jóvenes. Esta realidad ya fue observada en estudios previos realizados en nuestra institución ${ }^{(6)}$. Asimismo, en otros países no industrializados, las quemaduras también afectan predominantemente a pacientes jóvenes ${ }^{(7)}$. Esto genera un importante impacto económico a nivel de la sociedad. Las quemaduras representan años de vida perdidos y una carga económica muy importante debido a secuelas 


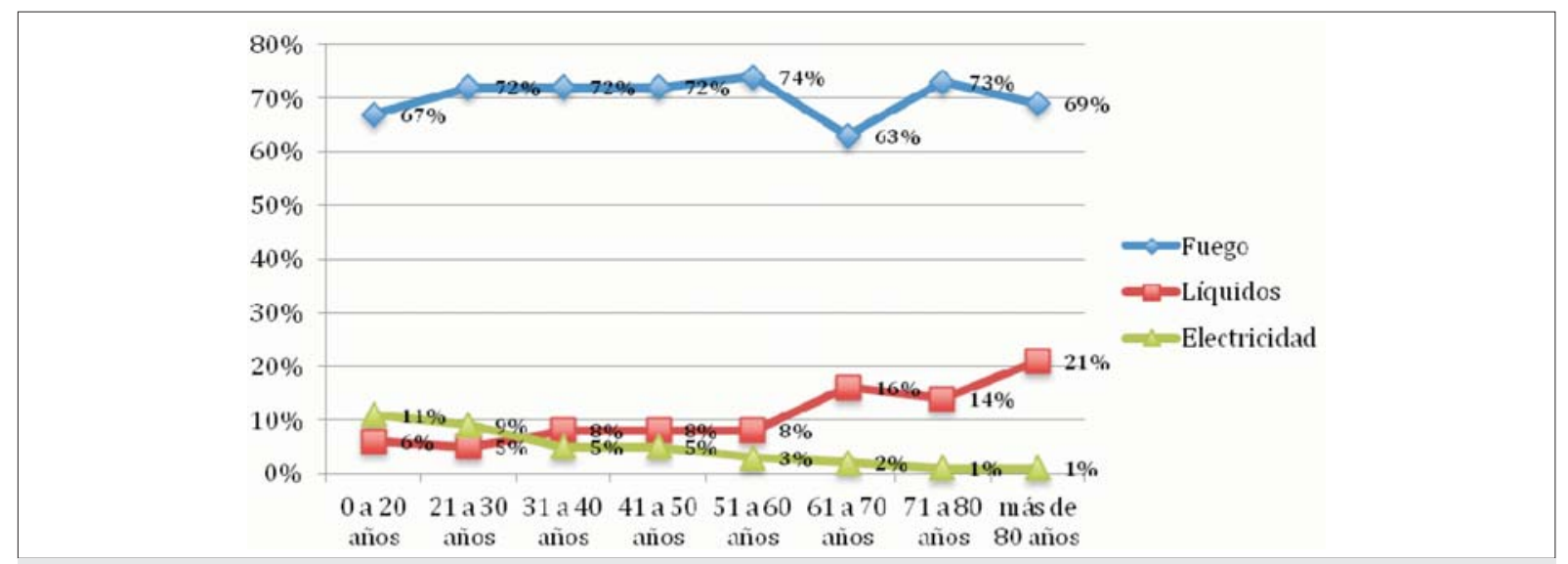

Figura 3. Agente causal de acuerdo al rango etario.

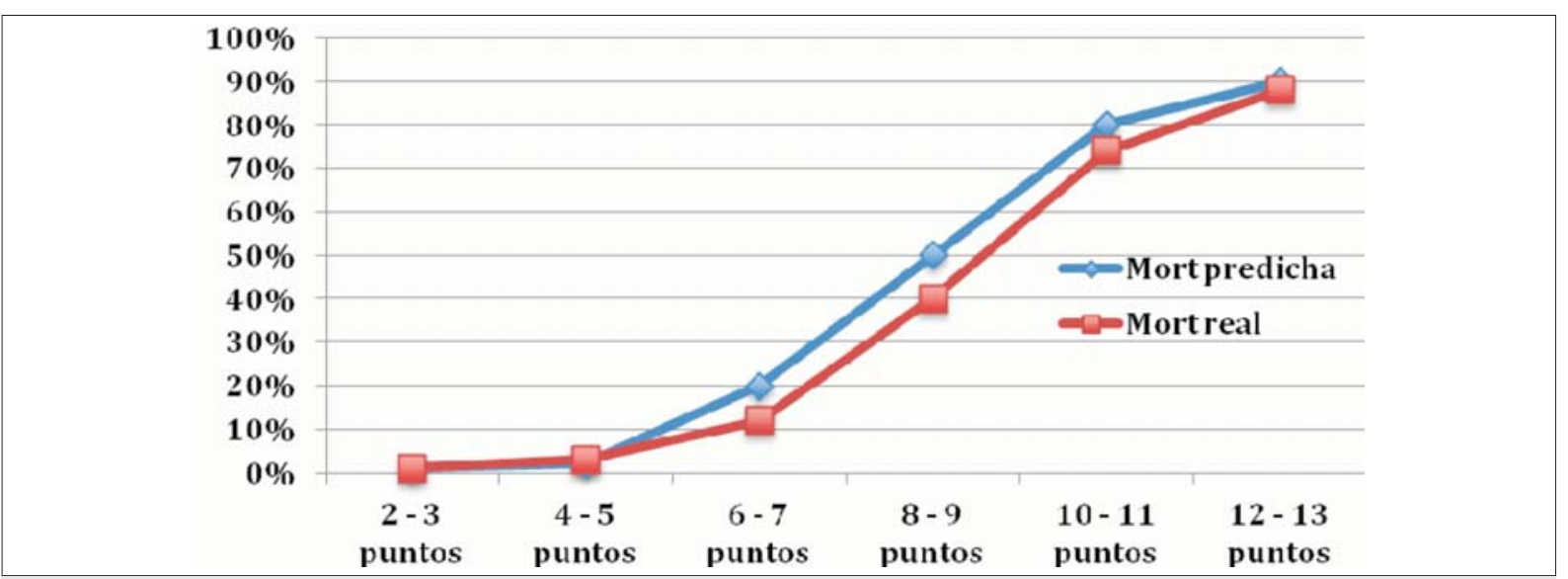

Figura 4. Mortalidad en función de la severidad de los pacientes determinada por el ABSI score.

e invalidez en personas en edad económicamente acti$\mathrm{va}^{(7)}$.

El agente generador de la quemadura difiere de acuerdo a la riqueza de la población. En países ricos predominan las quemaduras por líquidos calientes, mientras que en los países pobres predominan las quemaduras por fuego. Esta diferencia se observa incluso al comparar poblaciones de diferente nivel socioeconómico en un mismo país ${ }^{(8)}$. El estudio de Roberts, que evalúa las causas de muerte de niños de acuerdo a su nivel socioeconómico, encontró que las muertes por fuego son significativamente más frecuentes en la población más pobre. Esto está en estrecha relación con el uso del fuego para uso cotidiano, ya sea en la cocina, calefacción, etcéte$\mathrm{ra}^{(8)}$.

La estadía de los pacientes en nuestro centro fue de 16 días. Esta estadía es superior a la observada en otras patologías graves. El estudio de Bertullo y colaboradores, que analizó 153 pacientes en cinco UCI de Uruguay con sepsis y shock séptico, encontró una estadía de 12 días ${ }^{(9)}$. Varios estudios han analizado la estadía de los pacientes quemados, destacando que las mismas son más prolongadas que en otras patologías. Los diferentes trabajos evidencian estadías entre 1,8 y 2,6 días por cada $1 \%$ de quemadura ${ }^{(10,11)}$. Esto se vincula fundamentalmente a la necesidad de múltiples intervenciones quirúrgicas. La superficie quemada no es el único factor contribuyente para una mayor estadía, una mayor edad y un mayor número de comorbilidades también se asocian a estadías más prolongadas ${ }^{(10,11)}$.

La mortalidad de los pacientes ha descendido fundamentalmente en los centros de quemados ${ }^{(12)}$. El estudio de Mason y colaboradores, que analizó 772 pacientes quemados, puso en evidencia que la mortalidad de los pacientes asistidos en centros especializados descendió a lo largo del tiempo, en comparación con lo ocurrido en los centros no especializados ${ }^{(13)}$. Por otra parte, el momento del ingreso a los centros especializados también impacta en resultados. En este sentido, Ehrl y colaboradores compararon ingresos precoces versus tardíos a 


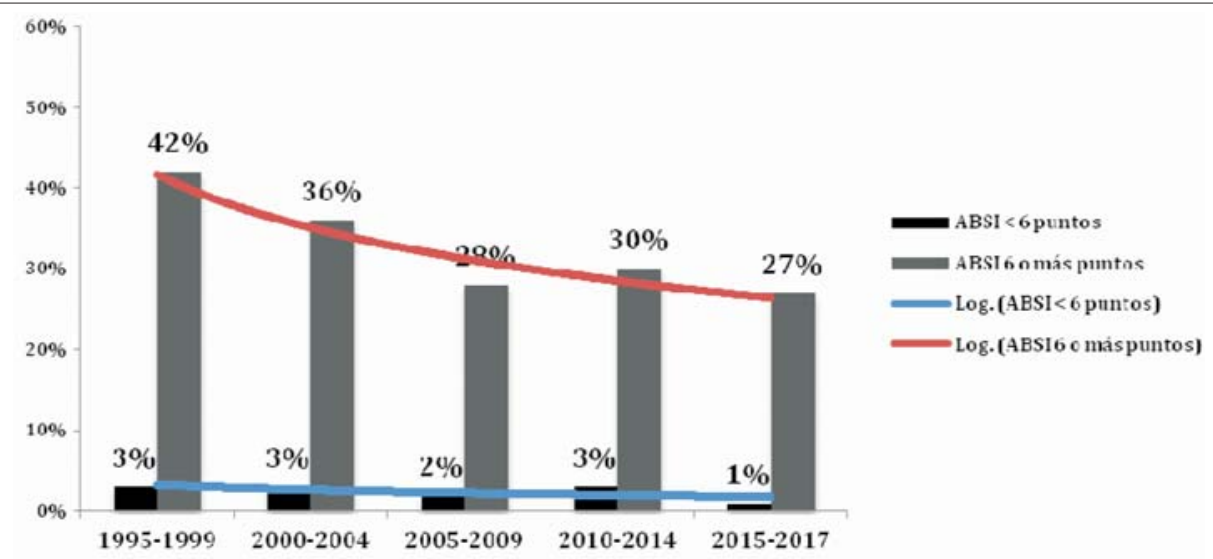

Figura 5. Evolución de la mortalidad de acuerdo a la severidad de los pacientes.

centros de quemados y evidenciaron una reducción en los días de asistencia respiratoria mecánica, estadía en la unidad y estadía hospitalaria en el grupo cuyo ingreso al centro se realizó en forma temprana ${ }^{(14)}$.

Si bien nuestro estudio no tuvo como objetivos evaluar las razones que generaron el descenso en la mortalidad de los pacientes, existen varias hipótesis tendientes a explicar este descenso de la mortalidad.

La especialización y el volumen de pacientes asistidos se asocian con mejores resultados. Esto se ha observado en diversas patologías y seguramente sea una de las razones asociadas al descenso de la mortalidad ${ }^{(15,16)}$.

Por otro lado, los avances en las diferentes especialidades implicadas en la asistencia del paciente gran quemado también juegan un rol en la mejora de los resultados. En este sentido, Vincent y colaboradores compararon 552 enfermos sépticos asistidos en UCI en el año 2002 versus 894 pacientes en el año 2012, encontrando mayor severidad y mayor sobrevida en los pacientes más recientemente asistidos ${ }^{(17)}$.

Otro factor probablemente implicado en la mejora de los resultados es el enfoque multidisciplinario que existe en los centros especializados, que logra evaluar los problemas de los pacientes en forma integral y no solamente desde la óptica de una especialidad.

Nuestro estudio presenta limitaciones. El uso de la base de datos electrónicos del centro implica que puede haber datos no registrados que alteren la severidad. Habitualmente este fenómeno genera una subestimación de la severidad, ya que los datos faltantes son considerados como normales. Por otra parte, pese a que existen formas de evaluación de la superficie quemada, la misma puede variar de acuerdo al observador y de esa forma alterar la categorización de severidad.

Finalmente, tan importante como conocer la mortalidad de los pacientes en el centro es conocer sus secuelas y calidad de vida, lo cual no es posible evaluar a través de los datos con que contamos a partir del sistema de información del centro.

\section{Conclusión}

Nuestro centro asiste a pacientes predominantemente jóvenes, con siniestros que involucran al fuego como el principal agente causal. La mortalidad en el centro es similar a la esperada de acuerdo a los scores de severidad, destacándose un descenso continuo de esta a lo largo de los años.

\section{Abstract}

Introduction: burned patients mortality has decreased because of a better understanding of initial shock pathophysiology, early surgical interventions, antibiotic therapy optimization and appropriate nutritional support. Mortality reduction has also been related to the creation of specialized burn centers. There are no recent reports of burn injury patients in our country.

Objective: to describe characteristics and evolution of patients assisted at the national burned center (Centro Nacional de Quemados, Uruguay).

Methods: transversal, observational, descriptive study. All patients admitted in the center with diagnosis of skin burns or inhalation injury, from the center's opening in 1995 through December 31, 2017.

Results: during the study period 3,050-burned patients were admitted, $25 \%$ of which had a total body surface area burnt of at least $20 \%$. There was a predominance of male sex (62\%) and young patients (more than $60 \%$ had less than 50 years old). The most frequent responsible agent was direct fire $(71 \%)$, followed by hot liquids $(9 \%)$ and electricity $(5 \%)$. Mechanical ventilation was needed in $43 \%$ of the patients, and mean length of stay in the center was 17 days. Overall mortality rate was 
$19.7 \%$, accordingly to that predicted by specific severity scores. In the sub-group of more severe patients, mortality has progressively decreased since the center opening.

Conclusions: our center assists young patients with burn injuries mostly caused by direct fire. Overall mortality is in line with the expected according to severity scores, having decreased progressively since the center inauguration.

\section{Resumo}

Introdução: a mortalidade dos pacientes com injúria térmica tem diminuído em todo o mundo devido, em parte, à criação de centros especializados no tratamento desta patologia. No nosso meio não existem estudos recentes que avaliem a evolução da mortalidade neste tipo de centro.

Objetivo: descrever as características da população atendida e a evolução da mortalidade no Centro Nacional de Quemados.

Métodos: estudo transversal, observacional, descritivo. Foram incluídos todos os pacientes admitidos com diagnóstico de queimadura cutânea ou de injúria inalatória desde a inauguração do Centro em 1995 até o dia 31 de dezembro de 2017.

Resultados: no período estudado, foram admitidos 3050 pacientes queimados, $25 \%$ apresentaram uma superfície corporal queimada maior ou igual a $20 \%$. Observou-se o predomínio de pacientes jovens (mais de $60 \%$ com menos de 50 anos) e de sexo masculino (62\%). $\mathrm{O}$ agente causal mais frequente foi o fogo direto $(71 \%)$, seguido por líquidos quentes $(9 \%)$ e eletricidade $(5 \%)$. $43 \%$ dos pacientes necessitaram assistência ventilatória mecânica, e a permanência média no Centro foi de 17 dias. A mortalidade global foi de $19,7 \%$, similar à esperada de acordo com os graus específicos de gravidade. A mortalidade dos pacientes mais graves vem diminuindo desde a abertura do centro.

Conclusões: nosso centro atende predominantemente pacientes jovem sendo o fogo o principal agente causal. A mortalidade é similar à esperada de acordo com os graus específicos de gravidade, destacando-se um descenso continuo da mesma ao longo dos anos.

\section{Bibliografía}

1. Peck MD. Epidemiology of burns throughout the world. Part I: Distribution and risk factors. Burns 2011; 37:1087-100.

2. Citron I, Amundson J, Saluja S, Guilloux A, Jenny H, Scheffer M, et al. Assessing burn care in Brazil: an epidemiologic, cross-sectional, nationwide study. Surgery 2018; 163(5):1165-72.
3. Mason SA, Nathens AB, Byrne J, Gonzalez A, Fowler R, Karanicolas $\mathbf{P}$, et al. Trends in the epidemiology of major burn injury among hospitalized patients: a population-based analysis. J Trauma Acute Care Surg 2017; 83:867-74.

4. Mason SA, Nathens AB, Byrne JP, Fowler RA, Karanicolas PJ, Moineddin R, et al. Burn center care reduces acute health care utilization after discharge: a population-based analysis of 1,895 survivors of major burn injury. Surgery 2017; 162:891-900.

5. Tobiasen J, Hiebert JM, Edlich RF. The abbreviated burn severity index. Ann Emerg Med 1982; 11:260-2.

6. Cabrera J, Baz M, Alzugaray P, Echavarría E, Vanerio J, Juri H. Epidemiología del Centro Nacional de Quemados del Uruguay. Rev Arg Quemaduras 2010; 1:13-9.

7. Davé DR, Nagarjan N, Canner JK, Kushner AL, Stewart BT. Rethinking burns for low \& middle-income countries: differing patterns of burn epidemiology, care seeking behavior, and outcomes across four countries. Burns 2018; 44(5):1228-34.

8. Roberts I. cause specific social class mortality differentials for child injury and poisoning in England and Wales. J Epidemiol Community Health 1997; 51:334-5.

9. Bertullo M, Carbone N, Brandes M, Silva M, Meiss H, Tejera $\mathbf{D}$, et al. Epidemiología, diagnóstico y tratamiento de la sepsis severa en Uruguay: un estudio multicéntrico prospectivo. Rev Méd Urug 2016; 32:177-89.

10. Cheng W, Yan-hua R, Fang-gang N, Wei-li D, Guo-an Z. Epidemiology of 1974 burn patients at a major burn center in Beijing: a nine-year study. J Burn Care Res 2012; 33:e228-33

11. Hosseini S, Rashtchi V, Kamali K, Moghimi M. Epidemiology and outcome of 2590 burned patients in Northwest Iran. Ann Burns Fire Disasters 2017; 30(2):85-90.

12. Sheridan RL. Burn care: results of technical and organizational progress. JAMA 2003; 290:719-22.

13. Mason SA, Nathens AB, Byrne J, Gonzalez A, Fowler R, Karanicolas $\mathbf{P}$, et al. Trends in the epidemiology of major burn injury among hospitalized patients: a population-based analysis. J Trauma Acute Care Surg 2017; 83:867-74.

14. Ehrl D, Heidekrueger P, Ninkovic M, Broer PN. Effect of primary admission to burn centers on the outcomes of severely burned patients. Burns 2018; 44(3):524-30.

15. Dres M, Austin PC, Pham T, Aegerter P, Guidet B, Demoule A, et al. Acute respiratory distress syndrome cases volume and ICU mortality in medical patients. Critical Care Med 2018; 46(1):e33-e40.

16. Lecuyer L, Chevret S, Guidet B, Aegerter P, Martel P, Schlemmer B, et al. Case volume and mortality in haematological patients with acute respiratory failure. Eur Respir J $2008 ; 32: 748-54$

17. Vincent JL, Lefrant JY, Kotfis K, Nanchal R, Martin-Loeches I, Wittebole X, et al. Comparison of European ICU patients in 2012 (ICON) versus 2002 (SOAP). Intensive Care Med 2018; 44:337-44. 